See Article page 241.

\section{Commentary: Lessons from the National Lung Screening Trial}

\author{
Daniel P. Raymond, MD
}

The National Lung Screening Trial was a landmark study and herculean effort that had profound clinical impact. ${ }^{1}$ One of the many benefits of the trial was a lengthy discourse on the evaluation and design of screening studies. The intent of a screening study is to decrease the disease-specific outcome of interest in an asymptomatic population. The population should carry the disease with a reasonable prevalence to optimize the efficiency of the screening exam and the positive predictive value of the study. The exam itself must be reliable, reproducible, accessible, and affordable. Potential harms must be carefully tracked and come in many forms, including further interventions required for a false-positive study as well as delays in diagnosis associated with a false-negative study. There are also concerns regarding overdiagnosis and overtreatment, both of which lead to more interventions and healthcare expenditures that have no benefit to the patient.

Into this fray step Akhtar-Danesh and colleagues ${ }^{2}$ by extending a former study ${ }^{3}$ that initially demonstrated a venous thromboembolic event (VTE) prevalence of $12.1 \%$ based on a 30-day screen that included computed tomography angiography and bilateral lower extremity duplex evaluation in a population of patients undergoing lung cancer resection. Notably, in that study, $20 \%$ of the VTE events were symptomatic, and thus this was not a pure screening population. The authors documented 30-day mortality rates of $5.2 \%$ for those experiencing an event and $0.64 \%$ in the entire population screened. In their subsequent study,

From the Departments of Thoracic and Cardiovascular Surgery, Cleveland Clinic, Cleveland, Ohio.

Disclosures: The author reported no conflicts of interest.

The Journal policy requires editors and reviewers to disclose conflicts of interest and to decline handling or reviewing manuscripts for which they may have a conflict of interest. The editors and reviewers of this article have no conflicts of interest.

Received for publication March 23, 2021; revisions received March 23, 2021; accepted for publication March 24, 2021; available ahead of print April 20, 2021.

Address for reprints: Daniel P. Raymond, MD, Associate Professor of Surgery,

Thoracic and Cardiovascular Surgery, Cleveland Clinic, 9500 Euclid Ave, J4-1,

Cleveland, OH 44195 (E-mail: Raymond3@ccf.org).

JTCVS Open 2021;6:246-7

2666-2736

Copyright (c) 2021 The Author(s). Published by Elsevier Inc. on behalf of The American Association for Thoracic Surgery. This is an open access article under the CC BY-NC-ND license (http://creativecommons.org/licenses/by-nc-nd/4.0/).

https://doi.org/10.1016/j.xjon.2021.03.018

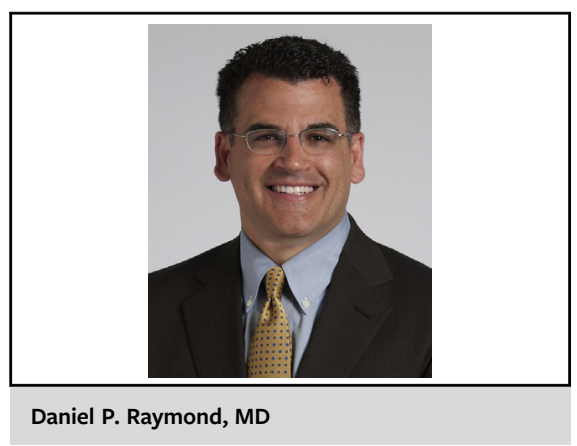

CENTRAL MESSAGE

The intent of a screening study is

to decrease the disease specific

outcome of interest in an

asymptomatic population.

Akhtar-Danesh and colleagues tracked this patient cohort out for a median of 3.6 years and found no difference in disease-free survival between lung cancer patients with VTE and those without VTE. Furthermore, they noted no difference in overall survival in a larger population that also included patients undergoing metastasectomy. Thus, they concluded that screening may be warranted in highrisk populations to improve survival.

Their results are interesting, and I must admit my bias in support of screening and prophylaxis following surgery for lung cancer. On the other hand, this study falls far short of providing convincing evidence in favor of VTE screening following lung cancer surgery. First and foremost, the authors have not demonstrated that screening decreases the disease-specific mortality rate of a screened population. Their demonstration that survival is equivalent when theoretically it should be worse is wholly inconclusive. This would require a control, unscreened population, which they do not have. Second, the populations are mixed and include symptomatic patients, which might actually bias the results against their conclusion. Furthermore, they have a mix of pathologies, including not only lung cancer, but also lung surgery for metastatic disease, thus further confounding the conclusion. Third, they have neglected one of the important rules of a screening study by selecting a contrasted computed tomography pulmonary arteriogram as one of the screening tools. This is a costly study with contrast and radiation exposure that may have limited accessibility. Finally, and importantly, they have not analyzed the potential harm associated with the screening study. This importantly would include bleeding events in 
patients being treated based on a positive screening test, renal failure associated with contrast exposure, and any other harm that occurred due to additional therapy or interventions, whether diagnostic or therapeutic.

I understand and laud the authors' efforts to raise awareness regarding the significant prevalence of VTE events following lung cancer surgery. This clearly is a problem that requires our attention, but in a logical and structured manner. Such a study would require a defined, higher-risk population and an acceptable screening test, such as lower extremity duplex examination. Patients would be randomized to screening or no screening and then would be followed out carefully for 5 years to determine diseasespecific and overall survival as well as to carefully evaluate for possible harm. A goal would need to be set for a set mortality reduction that would dictate the size of the population required. Such a study would be challenging to perform, given the large number of patients required and associated cost, as suggested by the 9-figure cost of the National Lung Screening Trial. ${ }^{4}$ Until such a study is completed, we all must remain vigilant and seek out opportunities to advance our understanding of this challenging problem.

\section{References}

1. The National Lung Screening Trial Research Team. Reduced lung-cancer mortality. $N$ Engl J Med. 2011;365:395-409.

2. Akhtar-Danesh G-G, Ben-Avi R, Agzarian J, Shargall Y. Is asymptomatic postoperative venous thromboembolism associated with long-term survival in patients undergoing lung resection for malignancy? JTCVS Open. 2021;162:241-5.

3. Agzarian J, Hanna WC, Schneider L, Schieman C, Finley CJ, Peysakhovich Y, et al. Postdischarge venous thromboembolic complications following pulmonary oncologic resection: an underdetected problem. J Thorac Cardiovasc Surg. 2016;151:992-9.

4. National Cancer Institute. Lung cancer screening saves lives: the National Lung Screening Trial. Available at: https://www.cancer.gov/research/progress/discov ery/nlst. Accessed March 1, 2021. 